\title{
CARACTERIZAÇÃO DA PRODUÇÃO E QUALIDADE FíSICO-QUÍMICA DE MÉIS PRODUZIDOS NO ESTADO DE PERNAMBUCO
}

\author{
João Luiz Aleixo Silva¹, Alexandre de Farias' ${ }^{1}$, Thaís de Souza Bovi², Ana Paula da \\ Silva Dib1 ${ }^{1}$, Lidia Maria Ruv Carelli Barreto ${ }^{1}$, Ricardo de Oliveira Orsi²
}

\author{
1 UNITAU \\ 2 UNESP - Botucatu \\ Correspondência: Ricardo de Oliveira Orsi: orsi@fmvz.unesp.br
}

\begin{abstract}
RESUMO: O presente estudo teve por objetivos contribuir com a caracterização da produção apícola no Estado de Pernambuco e avaliar a qualidade físico-química de méis produzidos na região. Para isto, aplicou-se um formulário dirigido ao órgão representativo da classe apícola sobre aspectos produtivos e técnicos dos apicultores. Foram obtidas 14 amostras de mel de abelhas Apis mellifera africanizadas, acondicionadas em recipientes plásticos estéreis e encaminhadas ao Laboratório de Controle de Qualidade de Produtos Apícolas (CEA-UNITAU). Observou-se que a maioria dos apicultores possui de 50 a 100 colmeias (57,14\%), 28,57\% de 100 a 200 colmeias e $14,28 \%$ mais de 500 colmeias, sendo que $85,71 \%$ produzem de 30 a $50 \mathrm{~kg} \mathrm{mel} / \mathrm{colmeia} /$ florada. Todos utilizam a colmeia padrão Langstroth e $85,71 \%$ adquirem seus enxames por meio de captura. Com relação à qualidade físico-química do mel, observou-se que os teores de umidade variaram de $18,2 \%$ a $22,0 \%$,

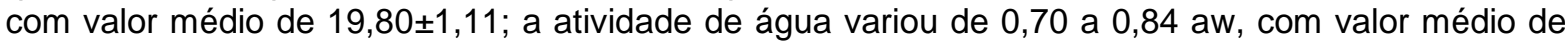
$0,79 \pm 0,05 \mathrm{aw}$; a acidez total foi de $24,91 \pm 8,99 \mathrm{meq} / \mathrm{kg}$ e $o$ índice médio de hidroximetilfurfural foi de $16,32 \pm 17,88 \mathrm{meq} / \mathrm{kg}$. Os resultados obtidos encontram-se dentro dos limites de qualidade estabelecidos pela legislação brasileira, exceto a atividade de água que excedeu o limite máximo de 0,65 aw. Os dados obtidos neste trabalho demonstram desenvolvimento da apicultura no Estado do Pernambuco e que o mel produzido apresenta boa qualidade.
\end{abstract}

Palavras-chave: apicultura; mel; produtividade; qualidade

\section{PRODUCTION AND PHYSICAL-CHEMICAL CHARACTERIZATION OF HONEY PRODUCED IN PERNAMBUCO STATE}

\begin{abstract}
The present study had as objective contribute to the characterization of beekeeping in the Pernambuco State and evaluate the physical-chemical quality of the honeys produced in the region. For this, was applied a directed formulary to the representative organ of beekeeping class about the productives and technicals aspects of beekeepers. Was obtained 14 samples of honey by Apis mellifera africanized, stored in sterile plastic vessels was sent to the Beekeeping Products Quality Control Laboratory (CEA-UNITAU). Was observed that the most of beekeepers have of 50 to 100 hives (57,14\%), $28,57 \%$ of 100 to 200 hives and $14,28 \%$ more than 500 hives, being that $85,71 \%$ produce 30 to $50 \mathrm{~kg}$ honey/hives/flowering. All use the standard hive Langstroth and $85,71 \%$ obtain their swarm by capture. About the physical-chemical quality of the honey, was observed that moisture content varied from $18,2 \%$ to $22,0 \%$, with mean value of $19,80 \pm 1,11$; the water activity varied from 0,70 to 0,84 aw, with mean value of $0,79 \pm 0,05$ aw; the total acidity was $24,91 \pm 8,99 \mathrm{meq} / \mathrm{kg}$ and the average index of hydroxymethylfurfural was $16,32 \pm 17,88 \mathrm{meq} / \mathrm{kg}$. The results obtained are according to the quality limits established by the brazilian legislation, excepted the water activity that exceeded the maximum limit of 0,65 aw. The datas obtained in this paper shows the development of beekeeping in Pernambuco State and the honey presents nice quality.
\end{abstract}

Key Words: beekeeping, honey, productivity, quality 


\section{INTRODUÇÃO}

A apicultura no Brasil foi iniciada no período de colonização, com a introdução das abelhas Apis mellifera mellifera pelo padre Antônio Carneiro, no Estado do Rio de Janeiro em 1839 (Wiese, 2005). Em seguida, imigrantes europeus instalaram nas regiões Sul e Sudeste, novos enxames de subespécies européias. Contudo, foi em 1956 que a apicultura brasileira teve seu marco principal, com a introdução da abelha africana (Apis mellifera scutellata) e a consequente africanização dos enxames em todo território nacional.

Desde então, a apicultura brasileira experimentou grande crescimento, na área produtiva, na pesquisa, no desenvolvimento de novos equipamentos e manejo adequados à nova realidade. Hoje, a apicultura está difundida em todas as regiões do Brasil, obtendo-se mel na Amazônia, Mata Atlântica, Pantanal, Caatinga, Pampa Gaúcho e Cerrado (Sabbag e Nicodemo, 2011). Na região nordeste, a apicultura teve um grande desenvolvimento, sendo Piauí, Ceará, Pernambuco e Bahia os estados da região que se destacam na produção de mel (Mendonça et al., 2006).

Segundo a Food Administration Organization (FAO, 2012), a produção mundial de mel é de 1.541.282 milhões de toneladas, e de acordo com o Instituto Brasileiro de Geografia e Estatística (IBGE) a produção nacional atingiu um volume de $38.017 .403 \mathrm{mil}$ toneladas de mel/ano. Desta, a região nordeste produziu $13.116 .528 \mathrm{mil}$ toneladas, correspondentes a $34,5 \%$ de toda produção nacional, percentual este que leva o nordeste a ocupar o segundo lugar entre as regiões produtoras de mel (IBGE, 2012).

O Estado de Pernambuco é hoje o $9^{\circ}$ maior produtor de mel do país e $04^{\circ}$ maior produtor da região nordeste, atingindo a produção de 2094 toneladas de mel no ano de 2010 (IBGE 2012).

Tamanha produtividade se deve a iniciativas públicas que pretendem minimizar impactos socioambientais na região do semiárido brasileiro. Neste sentido, a inserção da atividade apícola no Estado, surgiu como uma estratégia para o desenvolvimento sustentável do território, já que é uma atividade economicamente viável, socialmente justa e ecologicamente correta. A atividade apícola de Pernambuco está consolidada em toda sua extensão litoral, agreste e sertão (Mendonça et al., 2006).

Devido à produção melífera de Pernambuco destacar-se na região nordestina, é necessário que se caracterize o mel produzido, com 0 intuito de conferir melhorias e estabelecer garantias do produto final para fins de comercialização. É por meio de características físico-químicas que se pode conhecer o mel produzido em diferentes regiões. Para isso, algumas análises podem ser feitas, dentre elas destacam-se: teor de umidade, atividade de água, acidez total e hidroximetilfurfural (HMF) (Dische, 2008).

Neste sentido, o presente trabalho se propôs a contribuir para a caracterização da apicultura no Estado do Pernambuco e avaliar a qualidade físico-química do mel produzido na região.

\section{MATERIAL E MÉTODOS}

\section{Análise dos Principais Indicadores} Produtivos e Técnicos

A análise da produção apícola no Estado de Pernambuco foi realizada por meio da aplicação de um questionário dirigido ao órgão representativo da classe apícola, o qual foi respondido por 14 apicultores associados. O questionário pretendeu avaliar o uso de técnicas e equipamentos adequados 
Pernambuco

para a produção de mel; e ainda avaliar a produtividade dos apicultores. Os tópicos abordados no questionário foram: número de colmeias por apicultor, produção média de mel por colmeia/safra, meses de produção de mel, possíveis plantas forrageadas pelas abelhas, tipo de colmeia utilizada, material utilizado para fazer fumaça, se é feita a captura de enxames, divisão e união de família e o uso de tela excluidora entre o ninho e melgueira (ou sobreninho).

\section{Análise das características físico- químicas}

Foram obtidas 14 amostras de mel produzidas por abelhas Apis mellifera africanizadas, em apiários localizados no Estado de Pernambuco, no período de maio a julho de 2006, em apiários fixos, com florada predominante silvestre. As amostras de méis foram encaminhadas ao Laboratório de Controle de Qualidade de Produtos Apícolas do CEA-UNITAU para proceder às análises físico-químicas.

O índice Hidroxidometilfurfural (HMF) foi determinado através de leitura espectrofotométrica em onda de absorção a 550nm (Instituto Adolfo Lutz 1985).

A acidez total $(\mathrm{mEq} / \mathrm{kg})$ foi determinada mediante a titulação com $\mathrm{NaOH} 0,1 \mathrm{~N}$, seguindo-se as normas analíticas do Instituto Adolfo Lutz (1985).

As determinações de umidade foram efetuadas em refratômetro de Abbé, colocando-se uma gota de mel no prisma do aparelho e focalizando para a leitura, corrigindo-se a leitura para a temperatura padrão de $20^{\circ} \mathrm{C}$. A percentagem de umidade foi obtida pela relação do índice de refração com os valores encontrados na tabela de Chataway (Instituto Adolfo Lutz 1985).

$A$ atividade de água $\left(a_{w}\right)$ foi obtida em uma unidade psicrométrica termoelétrica DECAGON CX2OM
(Decagon Devices, Inc., Pullman, WA, USA). O aparelho foi calibrado com solução padrão saturada de nitrato de magnésio (Novasina, Kontroll, Tablete BAG TNr 54703).

\section{RESULTADOS E DISCUSSÃO}

Em relação ao número de colmeias, $57,14 \%$ dos apicultores possuem de 50 a 100 colmeias, 28,57\% de 100 a 200 colmeias e 14,28\% mais de 500 colmeias. Segundo Sebrae (2009), o número de colmeias presente em um apiário define a tipologia a ser designada para uma unidade produtiva, ou seja, a presença de até 20 colmeias caracteriza a apicultura familiar e acima de 20 colmeias como profissional.

A produtividade verificada por enxame foi de $30 \mathrm{~kg}$ a $50 \mathrm{~kg} /$ florada (85,71\% dos casos). Alves et al. (2006), analisando a produção de apiários nos municípios cearenses de Camocim, Carnaubal, Croata, Santana do Acaraú e Sobral, verificaram a produção de 17 $\mathrm{kg} \mathrm{mel/colmeia/ano.} \mathrm{De} \mathrm{acordo} \mathrm{com}$ Brito (2011) no Estado da Bahia existem aproximadamente 185 mil colmeias, sendo em média 33 colmeias por apicultor com produtividade média de 19 $\mathrm{kg}$ mel/ano.

O período de florescimento principal relatado por $42,86 \%$ dos apicultores compreende os meses de fevereiro a junho, sendo as espécies vegetais mais citadas para a produção de mel: Piptadenia moniliformis (angico de bezerro) Borreria verticilata (vassourinha-de-botão), Croton sonderianus (marmeleiro), Ipomoea sp. (jitirana), Hyptis suaveolens (bamburral), Seriania sp. (cipó-uva), Eriope hypenioides (canelinha), Anacardium occidentale (caju), Mimosa caesalpinifolia (sabiá), Eugenia uniflora (pitanga), Citrus sinensis (laranja), Spondias mombins (cajá) e Mangifera indica (manga). Entretanto, 28,57\% dos apicultores apontaram os meses de 
setembro a novembro como o principal período de florada, assim como os períodos de setembro à março e junho à outubro. Essa diversidade nos relatos sobre as floradas sugere a necessidade da elaboração de um calendário apícola que oriente os apicultores da região, principalmente para aqueles que praticam a apicultora migratória. Segundo Oliveira et al. (2004), a apicultura exige estudo do local do apiário e suas imediações, já que as abelhas necessitam de pasto com flores abundantes em néctar e pólen, tanto para manutenção do exame quanto para a produção apícola.

A colmeia padrão Langstroth é utilizada por todos os apicultores, sendo que $71,43 \%$ adquirem as caixas de terceiro e $28,57 \%$ as fabricam. Popularmente conhecida como colmeia "Americana", a colmeia padrão Langstroth é o tipo mais usual em todo mundo (Caione, 2011). Seus componentes são móveis (ninho, assoalho, tampa e quadros) permitindo o manejo racional das colmeias, ou seja, sem causar injúrias ao exame, sendo, portanto o tipo de colmeia recomendada pela Confederação Brasileira de Apicultura (CBA) (Sebrae, 2009).

A maioria $(85,71 \%)$ dos apicultores adquire seus enxames por meio de captura, utilizando caixas iscas ou captura direta. $O$ restante divide os enxames quando necessário, aumentando seus apiários gradualmente. De acordo com Leopoldino et al. (2002), a atividade apícola tradicional baseia-se na captura de enxames silvestres. Contudo, a opção por constituir um apiário por colmeias capturadas implica na heterogeneidade genética das colônias capturadas e a possibilidade dos enxames serem portadores de doenças e parasitas prejudiciais à sanidade das abelhas, os quais podem afetar significativamente a produtividade das colônias (Silva e Freitas, 2004). Porém no presente trabalho, mesmo havendo alta percentagem de colmeias capturadas, foi verificada boa produtividade melífera.

O substrato mais utilizado para produção de fumaça foram folhas de eucalipto $(57,14 \%)$. Outros materiais como cascas de amendoim e restos de cera de abelha também são utilizados. Segundo Sebrae (2009), o material a ser utilizado no fumegador deve ser de origem vegetal e livre de contaminantes, devendo proporcionar fumaça fria, densa e sem cheiro forte.

Todos os apicultores afirmaram não utilizar tela excluidora de rainha. A tela excluidora tem 0 objetivo de confinar a rainha no ninho da colmeia, impedindo-a que suba até a melgueira fazendo postura de novas crias (Rusig et al., 2002). A presença de ovos e larvas no mel pode implicar na baixa qualidade do produto final.

Dessa forma, os dados apontados nesse estudo demonstram o potencial para o desenvolvimento da apicultura na região, já que todos os apicultores encaixam-se na tipologia profissional e apresentam adequado conhecimento técnico, verificado pela boa produtividade dos enxames.

Em relação aos parâmetros físico-químicos das amostras coletadas de mel, observou-se que todas as características analisadas encontram-se dentro dos limites estabelecidos pela legislação, exceto a atividade de água.

Os resultados obtidos para o teor de umidade (\%), atividade de água $\left(\mathrm{a}_{\mathrm{w}}\right)$, HMF $(\mathrm{mg} / \mathrm{kg}$ ) e acidez total $(\mathrm{mEq} / \mathrm{Kg}$ ) nas amostras de mel analisadas encontram-se na Tabela 1.

Os valores médios de umidade encontrados nesse estudo assemelhamse a valores obtidos por outros autores. Araújo et al. (2006), encontraram o teor médio de umidade em $19,16 \%$ para 10 amostras de mel da cidade do CratoCE. Sodré et al. (2007) analisaram 20 amostras de mel do Estado do Ceará e 
Pernambuco

obtiveram $18,73 \%$ de umidade média. Abadio Finco et al. (2010), analisaram 24 amostras de mel da região sul do Estado do Tocantins e verificaram teor médio de umidade $18,9 \%$.

\begin{tabular}{|c|c|c|}
\hline Parâmetro & Média & Limites (Brasil 2000) \\
\hline \multirow{2}{*}{$\begin{array}{c}\text { Teor de Umidade } \\
(\%)\end{array}$} & $19,80 \pm 1,11$ & \multirow[t]{2}{*}{20} \\
\hline & $(18,2-22,0)$ & \\
\hline \multirow{2}{*}{$\begin{array}{l}\text { Atividade de água } \\
\qquad\left(\mathrm{a}_{w}\right)\end{array}$} & $0,79 \pm 0,05$ & \multirow[t]{2}{*}{$0,5-0,65$} \\
\hline & $(0,70-0,84)$ & \\
\hline \multirow[t]{2}{*}{$\mathrm{HMF}(\mathrm{mg} / \mathrm{kg})$} & $14,21 \pm 4,21$ & \multirow[t]{2}{*}{ Máximo 60} \\
\hline & $(9,6-20,91)$ & \\
\hline \multirow{2}{*}{$\begin{array}{c}\text { Acidez total } \\
(\mathrm{mEq} / \mathrm{kg})\end{array}$} & $30,21 \pm 4,51$ & \multirow[t]{2}{*}{50} \\
\hline & $(22-40)$ & \\
\hline
\end{tabular}

A análise de umidade é indicativa da maturidade do mel, teores de umidade acima de $20 \%$ significa que o produto ainda está verde, correndo grande risco de fermentação (Sebrae, 2009). No presente estudo, o teor médio deste parâmetro encontra-se dentro do limite estabelecido pela legislação brasileira.

A umidade é o segundo componente em quantidade na composição do mel (15 a $20 \%$ ) e pode ser influenciada pela origem botânica da planta, por condições climáticas e geográficas ou pela colheita do mel antes da sua completa maturidade. Essa característica pode influenciar na viscosidade, peso específico, maturidade, cristalização, sabor, conservação e palatabilidade do mel (Santos et al., 2010). Segundo Filho et al. (2011), a umidade é uma característica determinante à sua qualidade, alguns microrganismos precisam de um mínimo de umidade para se desenvolver e alterar as características físico químicas, interferindo imediatamente na vida de prateleira desse produto.

A atividade de água (Aw) não é um parâmetro estabelecido pela legislação, porém, indica a possibilidade de desenvolvimento microbiano. Os parâmetros atividade de água e umidade, em quantidades altas, indicam uma menor vida útil do alimento (Schlabitz et al., 2010). Os valores para atividade de água variaram entre 0,70 a $0,84 a_{w}$, com valor médio de $0,79 \pm 0,05$ $a_{w}$ (Tabela 1). Bendini e Souza (2008) avaliando 24 amostras de mel de cajueiro no município de Cascavel, CE, encontraram o valor médio de $0,70 \mathrm{a}_{\mathrm{w}}$ para atividade de água. Denardi et al. (2005), observaram em méis comercializados no município de São Paulo que a atividade de água nas amostras analisadas variou de 0,49 à $0,66 a_{w}$. Schlabitz et al. (2010), encontraram valores de 0,54 à $0,62 a_{w}$ em 12 amostras de mel da região do Vale do Taquari/RS.

Franco (2002) estabelece que os valores de atividade de água $\left(a_{w}\right)$ mínima em alimentos, necessária para a multiplicação de bolores xirofíticos e leveduras osmofílicas são, respectivamente, 0,65 e 0,61 . Desta forma, verifica-se que as amostras de mel analisadas apresentam valor médio de atividade de água que propicia o crescimento de leveduras presentes no mel, com possível deterioração do produto.

O valor médio de acidez total foi de $30,21 \mathrm{meq} / \mathrm{kg}$, estando dentro dos limites apontados pela legislação (Tabela 1). Sodré et al. (2007), encontraram o valor médio de acidez total de $30,13 \mathrm{meq} / \mathrm{kg}$ em amostras de méis provenientes de diferentes municípios do Ceará. Bendini e Souza (2008) encontraram média de 30,21 \pm $4,51 \mathrm{meq} / \mathrm{kg}$ ao analisar $0 \mathrm{mel}$ de cajueiro (Anacardium occidentale L). Filho et al. (2011), encontraram valor médio $48,81 \mathrm{meq} / \mathrm{kg}$ de acidez total em amostras de méis provenientes da cidade de Pombal-PB.

Segundo Welke et al. (2008) méis que apresentam valores de acidez inferiores a 50,0 $\mathrm{meq} / \mathrm{kg}$ indicam ausência de fermentação, qualificando 
assim as amostras estudadas. A acidez do mel é atribuída a diversos fatores como fonte de néctar, atividade enzimática da glicoseoxidase que origina 0 ácido glucônico, ação de bactérias durante a maturação e minerais.

Em relação ao índice de HMF a média encontrada para as quatorze amostras foi de 14,21 \pm 4,21 mg/kg (Tabela 1), estando dentro do limite estabelecido pela legislação brasileira. Outros estudos encontraram valores superiores de HMF nos méis analisados, como os relatados por (Filho et al., 2011) 20,6mg/kg, (Sodré et al., 2007) $31,45 \mathrm{mg} / \mathrm{kg}$ e (Araújo et al., 2006) $96,87 \mathrm{mg} / \mathrm{kg}$. Considerando- se que alguns mercadores externos exigem níveis inferiores ao da legislação brasileira, é necessário que o mel recém coletado apresente níveis mínimos de HMF, para que possa manter sua qualidade por mais tempo (Lopes et al., 2011).

As análises de acidez e HMF são indicadoras de deterioração do mel, ou seja, avaliam o frescor do produto, processos de aquecimento e ainda tempo de armazenamento (Sebrae, 2009). No presente estudo os índices HMF e acidez total mantiveram-se dentro dos limites estabelecidos pela legislação, sugerindo boa qualidade do mel de Pernambuco.

\section{CONCLUSÃO}

Os dados relatados nesse trabalho demonstram que a apicultura no Estado do Pernambuco encontra-se em franco desenvolvimento. Essa afirmação sustenta-se pelo número de colmeias observadas por apicultor, pela média de produção colmeia/florada e ainda pela qualidade do mel analisado.

\section{REFERÊNCIAS}

ABADIO FINCO, F. D. B.; MOURA, L. L.; SILVA, I. G. Propriedades físicas e químicas do mel de Apis mellifera L. Ciência e Tecnologia de Alimento, Campinas, v.30, n.3, p.706-712, 2010.

ALVES, J. E.; PONTE, A. E.; GADELHA, J. V. $M$. et al. Análise da apicultura desenvolvida em cinco municípios da região norte do Estado do Ceará. In: ANAIS DO CONGRESSO BRASILEIRO DE APICULTURA, 16., 2006, Aracaju. Anais... Aracajú, 2006. CD-ROM.

ARAÚJO, D. R.; SILVA, R. H. D.; SOUSA, J. S. Análise físico-química do mel comercializado na cidade do Crato, CE. Revista de Biologia e Ciências da Terra, Campina Grande, v.6, n.1, p.51-55, 2006.

BENDINI, J. N.; SOUZA, D. C. Caracterização melissopalinológica, físico-química e sensorial do mel de abelhas Apis mellifera L.

(Hymenoptera: Apidae) proveniente da florada do cajueiro. Ciência Rural, Santa Maria, v.38, n.2, p.565-567, 2008.

BRASIL. Instrução Normativa ํㅡ 11, de 20 de outubro de 2000. Estabelece o regulamento técnico de identidade e qualidade do mel. Diário Oficial da República Federativa do Brasil, Brasília, 23 out. 2000. Seção 1, p.16-17.

BRITO, F. E. M. Agricultores, cooperativas e organização da produção de mel no território Nordeste II. In: CONGRESSO LUSO AFRO BRASILEIRO DE CIÊNCIAS SOCIAIS, 11, 2011, Salvador. Anais... Universidade Federal da Bahia, 2011.

CAIONE, G.; CAIONE, W.; SILVA, A. F. et al. Avaliação econômica da atividade apícola em Alta Floresta, MT: UM estudo de caso. Revista de Ciências Agro-Ambientais, Alta Floresta, v.9, n.1, p.59-69, 2011.

DENARDI, C. A. S.; NISHIMOTO, E. J.; BALIAN, S. C. et al. Avaliação da atividade de água e da contaminação por bolores e leveduras em mel comercializado na cidade de São Paulo, SP, Brasil. Revista Instituto Adolfo Lutz, v.64, n.2, p.219-222, 2005.

DISCHE, E. Color reactions of carbohydrates. In: WHISTLER, R. L.; WOLFRAM, M. L. (Ed.). Methods in carbohydrates chemistry. New York: Academic Press, 2008. v.1, p.477- 512.

FILHO, J. P. A.; MACHADO, A. V.; ALVES, F. M. S. et al. Estudo físico-químico de qualidade do mel de abelha comercializado no município de Pombal -PB. Revista Verde de 


\section{Agroecologia e Desenvolvimento}

Sustentável, Mossoró, v.6, n.3, p.83-90, 2011.

FOOD AND AGRICULTURE ORGANIZATION FAO. Estatísticas, 2012. Disponível em: <http://www.fao.org>. Acesso em: 06 jul. 2012.

FRANCO, B. D. G. M.; LANDGRAF, M. Microbiologia dos alimentos. Ed. Atheneu. 2002, 182 p.

IBGE. Instituto Brasileiro de Geografia e Estatística. Disponível em:< http://www.ibge.gov.br/>. Acesso em: 06 de jul. 2012.

\section{INSTITUTO ADOLFO LUTZ. Normas}

Analíticas do Instituto Adolfo Lutz: Métodos químicos e físicos para análise de alimentos. v.1, 3.ed., São Paulo, 1985.

LEOPOLDINO, M. N.; FREITAS, B. M.; SOUSA, R. M. et al. Avaliação do feromônio de Nasanov sintético e óleo essencial de capim santo (Cymbopogon citratus) como atrativos para enxames de abelhas africanizadas (Apis

mellifera). Ciência Animal, Fortaleza, v.12, n.1, p.19-23. 2002.

LOPES, M. T. R.; BARBOSA, A. L.; NETO, J. M. V. et al. Alternativas de sombreamento para apiários. Pesquisa Agropecuária Tropical, Goiânia, v.41, n.3, p.229-305, 2011.

MENDONCA, S. A; RAMOS, S. A. V., MOURA, M. G. S. Apicultura, uma alternativa economicamente viável para pequenos agricultores da região do semi-árido pernambucano. In: ANAIS DO CONGRESSO BRASILEIRO DE APICULTURA, 16, 2006, Aracaju. Anais... Aracajú, 2006. 1 CD-ROM.

OLIVEIRA, F.; COSTA, S. M. A. L.;

TARSITANO, M. A. A. et al. Produção de mel na região noroeste do Estado de São Paulo: um estudo de caso de produtor familiar. Informações Econômicas, São Paulo, v.34, n.2, p.15-24, 2004.

RUSIG, A.; NOGUEIRA-COUTO, R. H.; COUTO, L. A. Tela excluidora de rainha na produção de mel e na longevidade das operárias em colmeias de Apis mellifera. Ciência Rural, Santa Maria, v.32, n.2, p.329-334, 2002.

SABBAG, O. J.; NICODEMO, D. Viabilidade econômica para produção de mel em propriedade familiar. Pesquisa Agropecuária Tropical, Goiânia, v.41, n.1, p.94-101, 2011.

SANTOS, D.C.; MARTINS, J.N.; SILVA, K.F.N.L. Aspectos físico-químicos e microbiológicos do mel comercializado na cidade de Tabuleiro do norte do Ceará. Revista Verde de

\section{Agroecologia e Desenvolvimento}

Sustentável, Mossoró, v.5, n.1, p.79-85, 2010.

SCHLABITZ, C.; SILVA, S.A.F.; SOUZA, C.F.V. Avaliação de parâmetros físico-químicos e microbiológicos em mel. Revista Brasileira de Tecnologia Agroindustrial, v.4, n.1, p.80-90, 2010.

SEBRAE. Manual de boas práticas apícolas Campo. Série qualidade e segurança dos alimentos, 2009. Disponível em: $<$ http//:www.sebrae.com.br.>. Acesso em 04 jul. 2012.

SILVA, R. H. D.; FREITAS, B. M. Produção e desenvolvimento de colônias de abelhas africanizadas (Apis mellifera) a partir de diferentes áreas e idades de cria. Ciência Rural, Santa Maria, v.34, n.2, p.545-549, 2004.

SODRÉ, G. S.; MARCHINI, L. C.; MORETI, A. C. C. C. et al. Caracterização físico-química de amostras de méis de Apis mellifera $\mathrm{L}$.

(Hymenoptera: Apidae) do Estado do Ceará. Ciência Rural, Santa Maria, v.37, n.4, p.11391144, 2007.

WELKE, J.E.; REGINATTO, S.; FERREIRA, D. et al. Caracterização físico-química de méis de Apis mellifera L. da região noroeste do Estado do Rio Grande do Sul. Ciência Rural, Santa Maria, v.38, n.6, p.1737-1741, 2008.

WIESE, H. Apicultura Novos Tempos. 2.ed. Guaíba-RS, 2005. 378p. 